\title{
THE BIFURCATION OF PERIODIC SOLUTIONS IN THE HODGKIN-HUXLEY EQUATIONS*
}

\author{
BY \\ WILLIAM C. TROY \\ University of Pittsburgh
}

\begin{abstract}
We consider the current clamped version of the Hodgkin-Huxley nerve conduction equations. Under appropriate assumptions on the functions and parameters we show that there are two critical values of $I$, the current parameter, at which a Hopf bifurcation of periodic orbits occurs.

Comparisons are made with numerical and experimental calculations of other authors in order to give a reasonable conjecture as to the global behavior of the families of bifurcating periodic orbits.
\end{abstract}

Introduction. In this paper we continue the analysis begun in [13] on the current clamped version of the Hodgkin-Huxley nerve conduction equations.

The Hodgkin-Huxley equations have the form

$$
\begin{aligned}
\frac{1}{R} \frac{\partial^{2} v}{\partial x^{2}}=C \frac{\partial v}{\partial t}+g_{k}(n)(v & \left.-v_{k}\right)+g_{1}(m) g_{2}(h)\left(v-v_{N a}\right)+\dot{g}_{l}\left(v-v_{l}\right), \\
\frac{\partial m}{\partial t} & =\gamma_{m}(v)\left(m_{\infty}(v)-m\right), \\
\frac{\partial n}{\partial t} & =\gamma_{n}(v)\left(n_{\infty}(v)-n\right), \\
\frac{\partial h}{\partial t} & =\gamma_{h}(v)\left(h_{\infty}(v)-h\right),
\end{aligned}
$$

The right-hand side of (1.1) represents the net current $I_{m}$ flowing across the membrane of the axon. The unknown functions are $v(x, t), m(x, t), n(x, t)$, and $h(x, t)$ where $t \geq 0$ and $-\infty<x<\infty . v$ represents the potential difference across the membrane of the axon at point $x$ and time $t$. The dimensionless variables $m$ and $h$ are called the sodium activation and deactivation variables, respectively. They control the switching on and off of the conductance of the membrane to sodium ions. Similarly, the dimensionless variable $n$ controls the activation of the potassium conductance. The parameters $R$ and $C$ denote the axon radius and capacitance, respectively. We assign $C$ the experimentally-determined value of $1 \mu \mathrm{fd}$. The remaining functions and parameters appearing in (1.1)-(1.4) are taken as known and the exact values assigned to them by Hodgkin and Huxley are found in [7].

A description of the original experiments of Hodgkin and Huxley and a derivation of the system (1.1)-(1.4) also appear in [7].

* Received May 13, 1976; revised version received May 19, 1977. This research was partially supported by NIH Grant no. NS 12457-01. 
The current clamped version of the Hodgkin-Huxley equation is the topic of interest in this paper and we now give a brief description. First, a long thin silver wire, called a space clamp, is inserted into the interior of the axon and along its entire length. This ensures that the potential difference $v$ across the membrane is independent of $x$. Thus $v$ depends only on $t$ and the system (1.1)-(1.4) becomes

$$
\begin{aligned}
\frac{d v}{d t}=-\left(g_{k}(n)\left(v-v_{k}\right)+g_{1}(m) g_{2}(h)\left(v-v_{N a}\right)+\bar{g}_{l}\left(v-v_{l}\right)\right), & \frac{d m}{d t}=\gamma_{m}(v)\left(m_{\infty}(v)-m\right), \\
\frac{d n}{d t} & =\gamma_{n}(v)\left(n_{\infty}(v)-n\right), \\
\frac{d h}{d t} & =\gamma_{h}(v)\left(h_{\infty}(v)-h\right),
\end{aligned}
$$

Next, with the space clamp in place and the nerve at rest (i.e., $v(0)=0, m(0)=m_{\infty}(0), n(0)$ $\left.=n_{\infty}(0), h(0)=h_{\infty}(0)\right)$, a positive current $I$ is applied across the membrane of the axon. Mathematically we can model this by

$$
\frac{d v}{d t}=I-\left(g_{k}(n)\left(v-v_{k}\right)+g_{1}(m) g_{2}(h)\left(v-v_{N a}\right)+\bar{g}_{l}\left(v-v_{l}\right)\right) .
$$

(Recall that the right-hand side of (1.1) represents the net current flowing across the membrane of the axon.)

The system (1.6)-(1.9) is called the current clamped version of the Hodgkin-Huxley equations. Several numerical investigations of (1.6)-(1.9) have been made by various authors and we give a brief description below of the results obtained.

With the nerve initially at rest Cole, Antosiewicz and Rabinowitz discontinually raise $I$ [1]. If $0<I<2.231 \mu \mathrm{amp}$. then the system merely returns to rest since the stimulus is not sufficient to trigger the formation of a large-amplitude pulse solution. However, once $I$ exceeds $2.231 \mu \mathrm{amp}$. a large-amplitude pulse solution is accomodated by the equations. This phenomenon continues to occur for larger values of $I$ until at some value between $5.25 \mu \mathrm{amp}$. and $6.0 \mu \mathrm{amp}$. repetitive firing occurs and a second pulse is observed before the system returns to rest. At $I=6.0 \mu \mathrm{amp}$. three pulses appear. For $I$ between $7.5 \mu \mathrm{amp}$. and $15.0 \mu \mathrm{amp}$. they compute what appears of be an infinite train of pulses. FitzHugh and Antosiewicz [4] repeated the aforementioned calculations after finding and correcting a slight error in the earlier program. Their new calculations agree to within a few percent. One discrepancy is that at $I=6.0 \mu \mathrm{amp}$. they compute only two pulses. However, as $I$ increases past $7.5 \mu \mathrm{amp}$. they also compute what appears to be an infinite train of impulses.

Cooley, Dodge and Cohen [2] investigated a suggestion of FitzHugh that repetitive firing in the space clamped axon may be associated with instability of the steady state solution. For each $I \geq 0$ their numerical calculations indicate that (1.6)-(1.9) has exactly one steady-state solution, $\pi_{I}=\left(v_{I}, m_{\infty}\left(v_{I}\right), n_{\infty}\left(v_{I}\right), h_{\infty}\left(v_{I}\right)\right)$. Let $A_{I}$ denote the Jacobian matrix of (1.6)-(1.9) evaluated at $\pi_{I}$. They found that the real parts of exactly two of the four eigenvalues of $A_{I}$ became positive as $I$ increases past $9.8 \mu \mathrm{amp}$. Thus there is a loss of stability of the steady state as $I$ passes through $9.8 \mu \mathrm{amp}$. The steady-state solution remains unstable for larger values of $I$ until $I$ passes through 154 and the real parts of all 
four eigenvalues become negative once again. Thus for $I$ between 9.8 and 154 one might expect that small perturbations from $\pi_{I}$ can grow in time and oscillations appear. In addition, for $I$ in a small interval to the left of 9.8 where $\pi_{I}$ is stable they computed what appears to be an unstable train of half-sized pulses. The results which we obtain in this paper give an explanation for the existence of these half-sized impulses.

Sabah and Spangler [11] also investigated the interval $6.8 \leq 9.8$ where the steady state is stable. For each $I$ in this interval they compute a large-amplitude periodic solution which appears to be stable. In addition, for $I \geq 9.8$ this family of periodic orbits continues to exist. However, as $I$ approaches 154 from below the amplitudes decrease and the periodic solutions disappear at $I=154$.

In this paper we use the Hopf theory of bifurcation of periodic solutions, and show that there is a second bifurcation point in addition to the one proven to exist by Troy ([12, 13]). Using these results we then present conjectures which unify all of the numerical evidence cited above and give a description of the global behavior of families of periodic solutions of (1.6)-(1.9).

In the next section we state several assumptions on the functions and parameters appearing in (1.6)-(1.9). These assumptions, based upon numerical evidence or physical reasoning, are necessary to rigorously prove our main results.

Sec. 3 consists of a statement of our main results. In the comment section following the statement of our main results we unify the numerical results described above with the results obtained in this paper. Also we state how our results might apply to explain smallamplitude oscillations which have been observed in cardiac purkinje fibres by Hauswirth, Noble and Tsien [6].

2. Assumptions on the Hodgkin-Huxley equations. As pointed out by FitzHugh [3], $n$ and $h$ change slowly with respect to $v$ and $m$. Thus, following Hastings [5], we multiply the right-hand side of (1.3) and (1.4) by a small positive parameter, $\epsilon$, and consider the system

$$
\begin{aligned}
\dot{v} & =I-F(v, m, n, h) \\
\dot{m} & =\gamma_{m}(v)\left(m_{\infty}(v)-m\right) \\
\dot{n} & =\epsilon \gamma_{n}(v)\left(n_{\infty}(v)-n\right) \\
\dot{h} & =\epsilon \gamma_{h}(v)\left(h_{\infty}(v)-h\right)
\end{aligned}
$$

where

$$
F(v, m, n, h)=g_{k}(n)\left(v-v_{k}\right)+g_{1}(m) g_{2}(h)\left(v-v_{N a}\right)+\bar{g}_{l}\left(v-v_{l}\right) .
$$

Our first three assumptions are similar to those found in ([5], [12], [13]):

(i) $v_{k}<v_{l}<v_{N a}$ and $v_{k}<0<v_{N a}$.

(ii) $g_{1}, g_{2}, g_{k}$, are analytic and non-negative on $[0,1], g_{1}{ }^{\prime}, g_{2}{ }^{\prime}, g_{k}{ }^{\prime}>0$ on $(0,1)$ and $g_{2}(0)$ $=0$.

(iii) $\gamma_{m}, \gamma_{n}, \gamma_{h}, m_{\infty}, n_{\infty}, h_{\infty}$ are analytic and positive on $(-\infty, \infty) ; m_{\infty}^{\prime}>0, n_{\infty}{ }^{\prime}>0$, $h_{\infty}{ }^{\prime}<0$ on $(-\infty, \infty) ; m_{\infty}(+\infty)=n_{\infty}(+\infty)=h_{\infty}(-\infty)=1, m_{\infty}(-\infty)=n_{\infty}(-\infty)=h_{\infty}(+\infty)$ $=0$.

The original functions and values of $v_{k}, v_{N a}$ and $v_{l}$ used by Hodgkin and Huxley all satisfy (i), (ii), and (iii).

Next we briefly state three additional assumptions necessary for the proof of our main 
results. These assumptions were used by the author in previous work ([12], [13]) and are made on the basis of numerical evidence and physical reasoning.

Let $D>0$ such that $v_{N a}-D>0$ and define the set

$$
\begin{gathered}
Q_{1}=\left\{(v, n, h) \mid 0 \leq v \leq v_{N a}-D, n_{\infty}(0) \leq n \leq n_{\infty}\left(v_{N a}-D\right),\right. \\
\left.h_{\infty}\left(v_{N a}-D\right) \leq h \leq h_{\infty}(0)\right\} .
\end{gathered}
$$

Define the function

$$
b(v, n, h)=F_{v}\left(v, m_{\infty}(v), n, h\right)+m_{\infty}{ }^{\prime}(v) F_{m}\left(v, m_{\infty}(v), n, h\right)
$$

where $(v, n, h) \in Q_{1}$.

From the numerical calculations of FitzHugh [3] we assume

$$
b\left(0, n_{\infty}(0), h_{\infty}(0)>0 .\right.
$$

The next assumption derives from the observation that during the initiation of a nerve impulse in response to an external stimulus there occurs a rapid increase in sodium conductance. From Eq. (1) and (2) it follows that the sodium conductance increases with $m$, and $m$ can increase rapidly only if $m_{\infty}{ }^{\prime}$ is large on some small interval of the form $[0, \delta]$. In addition, if $m_{\infty}{ }^{\prime}$ is large over $[0, \delta]$ then the function $b\left(v, n_{\infty}(v), h_{\infty}(v)\right)$ will change sign on $[0, \delta]$. Using the original functions of Hodgkin and Huxley, one can easily show that $b\left(v, n_{\infty}(v), h_{\infty}(v)\right)$ changes sign between $v=3$ and $v=4$. Thus we assume

(v) There is a first $\bar{v} \in\left(0, v_{N a}-D\right)$ where

$$
b\left((\bar{v}), n_{\infty}(\bar{v}), h_{\infty}(\bar{v})\right)=0
$$

and

$$
\frac{d b}{d v}\left(v, n_{\infty}(v), h_{\infty}(v)\right) \mid v=v<0
$$

From assumption (ii) and (2.7) we can show that

$$
\lim _{v \rightarrow v_{N} a} b\left(v, n_{\infty}(v), h_{\infty}(v)\right)>0 .
$$

Therefore there is a first $\hat{v} \in\left(\dot{v}, v_{N a}\right)$ such that

$$
b\left(\bar{v}, n_{\infty}(\hat{v}), h_{\infty}(\hat{v})\right)=0,
$$

and therefore,

$$
\left.\frac{d b^{\left(v, n_{\infty}(v), n_{\infty}(v)\right.}}{d v}\right|_{v=\hat{v}} \geq 0
$$

We assume

$$
\left.\frac{d b^{\left(c, n_{* i}\left(v, h_{x^{\prime}}(v) \mid\right.\right.}}{d v}\right|_{v=\hat{v}}>0
$$

It follows from $(2.1)-(2.5)$ that there is a steady-state solution $\Pi_{I}$ of $(2.1)-(2.4)$ corresponding to each solution of

$$
I-F\left(v, m_{\infty}(v), n_{\infty}(v), h_{\infty}(v)\right)=0 .
$$


In the squid axon there is only one resting level for a given $I \geq 0$. This physical reasoning, and numerical calculations (see Fig. 1) leads us to assume

(vii) For each $I \geq 0$ there is a unique $v_{I}$, continuously differentiable and increasing in $I$, which satisfies (2.13).

Also, from (2.13) it follows that for each $v \geq 0$ there is a unique $I(v)=F\left(v, m_{\infty}(v)\right.$, $\left.n_{\infty}(v), h_{\infty}(v)\right)$ which satisfies $(2.13)$. Therefore, we conclude that $v_{I(\bar{v})}=\bar{v}$ and $v_{I(\hat{v})}=\hat{v}$. For ease of notation we set $\bar{I}=I(\bar{v})$ and $\hat{I}=I(\hat{v})$.

To complete our mathematical preliminaries we need the following technical lemma.

Lemma 1. There is an $\alpha>0$ (independent of $\epsilon$ ) such that $\bar{v}+\alpha \leq \hat{v}-\alpha$ and with the additional properties

(a) $b\left(v, n_{\infty}(v), h_{\infty}(v)\right)<0$ for all $v \in(\bar{v}, \bar{v}+\alpha) \cup(\hat{v}-\alpha, \hat{v})$, and

(b) for each $v \in(\bar{v}, \bar{v}+\alpha) \cup(\hat{v}-\alpha, \hat{v})$,

$$
\left.\left(\gamma_{m}\left(\gamma_{n}+\gamma_{h}\right)\left(F_{v}+m_{\infty}{ }^{\prime} F_{m}\right)+\gamma_{n} n_{\infty}{ }^{\prime} F_{n}+\gamma_{h} h_{\infty}{ }^{\prime} F_{h}\right)\right|_{(m, n, h)=\left(m_{\infty}(v), n_{\infty}(v), h_{\infty}(v)\right.}>0
$$

Proof: We first note from (2.5) and assumptions (i)-(iii) that

$$
F_{v}>0, F_{m}<0, F_{n}>0, F_{h}<0
$$

for all $v<v_{N a}$, and $0<m, n, h<1$.

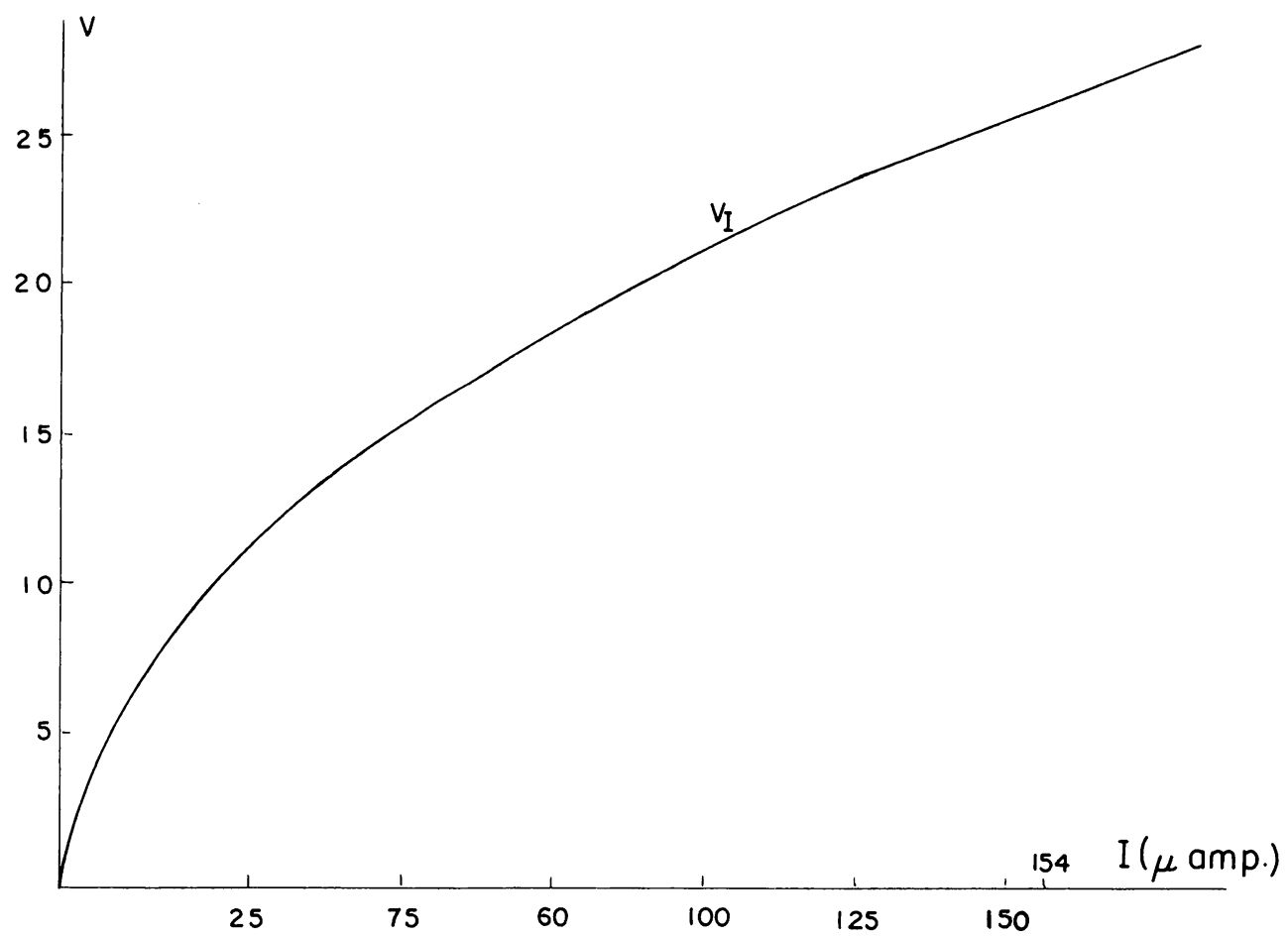

FIG. I. The numerical calculations of Cooley, Dodge and Cohen [2] indicate that for each $I>0$ there is a unique $v_{1}>0$ (increasing with $\left.I\right)$ such that $\left(v_{l}, m_{\infty}\left(v_{l}\right), n_{\infty}\left(v_{l}\right), h_{\infty}\left(v_{l}\right)\right)$ is the steady-state solution of $(2.1)-(2.4)$. Assumption (vi) is made on the basis of these calculations. 
The proof of parts (a) and (b) then easily follow from assumptions (i)-(iii), (2.8), (2.9), (2.11), (2.12) and (2.16).

3. Statement of main results. Let $I^{*} \in(-\infty,+\infty)$. We say that there occurs a bifurcation of periodic orbits from the steady state as $I$ passes through $I^{*}$ if there is an interval $U$ of the form $U=\left(I^{*}-\delta, I^{*}\right), U=\left(I^{*}, I^{*}+\delta\right)$ or $U=\left\{I^{*}\right\}$ such that for each $I \in U$ there is a periodic solution of $(2.1)-(2.4)$ whose amplitude approaches zero as $I$ approaches $I^{*}$ through $U$. In the special case that $U=\left\{I^{*}\right\}$ then $\pi_{I}{ }^{*}$ is a center for the system (2.1)-(2.4).

THEOREM: Recall the definitions of $I(v), I$ and $\hat{I}$ given in the previous section. There is an $\epsilon_{1}>0$ such that if $0<\epsilon<\epsilon_{1}$ then there exist positive numbers $I_{\epsilon}$ and $I^{\epsilon}$ with

$$
I<I_{\epsilon}<I^{\epsilon}<\hat{I}
$$

and such that as $I$ passes through $I_{\epsilon}\left(\right.$ or $\left.I^{\epsilon}\right)$ there occurs a bifurcation of periodic solutions of (2.1)-(2.4) from the steady-state solution $\pi_{I_{\epsilon}}\left(\right.$ or $\left.\pi_{I} \epsilon\right)$.

In this section we unify our bifurcation results with the numerical calculations described in Sec. 1 in order to give a conjectured diagram for the global behavior of a family of periodic orbits bifurcating from the steady state (see Fig. 2).

The results of Cole, Antosiewicz and Rabinowitz [1] and Cooley, Dodge and Cohen [2] indicate that for each $I \in(6.8,9.8)$ there is a stable large-amplitude periodic orbit, and an unstable small-amplitude periodic solution which disappears at $I=9.8$. Thus, in

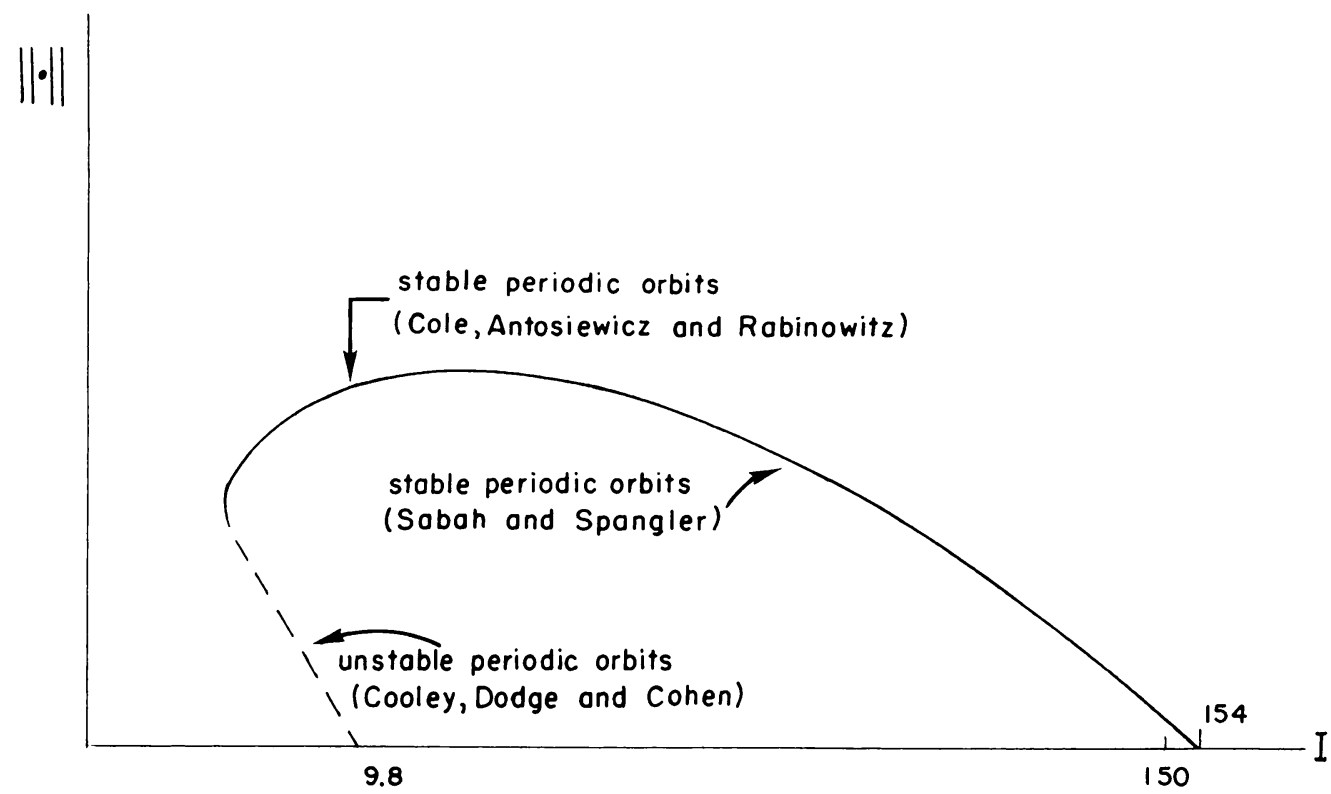

FIG. 2. The solid lower curve in the interval $6.8 \leq I \leq 9.8$ indicate the amplitudes of the unstable periodic orbits. The upper dotted curve indicates the amplitude of the unstable periodic orbits. The upper dotted curve indicates the amplitude of the stable oscillations observed by Sabah and Spangler, and Cole, Antosiewicz and Rabinowitz Bifurcation occurs to the left at $I=9.8 \mu \mathrm{amp}$. and $I=154 \mu \mathrm{amp}$. 
accordance with our theorem, it appears that there is a bifurcation of small-amplitude unstable periodic solutions on the interval $(6.8,9.8)$ with $I=9.8$ being the bifurcation point.

These unstable periodic orbits grow in amplitude as $I$ decreases until, at $I=6.8$, this family coalesces with the family of large amplitude stable solutions which exist for each $I$ $\in(6.8,9.8)$. It apperas that, at $I=6.8$, there is a unique periodic orbit which is semistable. As $I$ increases past 9.8 the family of large-amplitude stable periodic solutions computed by Sabah and Spangler [11] continues to exist. However, for considerably larger $I$ their amplitudes begin to decrease until, at $I=154$, these stable oscillations disappear altogether. Thus $I=154$ appears to correspond to the upper bifurcation point predicted by our theorem.

It appears that the upper bifurcation point and its associated family of small stable oscillations have not been previously observed in an actual squid axon. However, in their experiments on cardiac purkinje fibres, Hauswirth, Noble, and Tsien [6] have observed unexpected small-amplitude oscillations for various values of applied current. On the basis of experimental data they have modified the original Hodgkin-Huxley equations into a system which models the electrical behavior across the membrane of the purkinje fibre [10]. We conjecture that the bifurcation approach presented here might apply to their system and shed new light on the small oscillations which they have observed experimentally.

4. Proof. The main tool we use in the proof of our theorem is the Hopf theory of bifurcation of periodic solutions [8]. We use the translation as stated by $\mathrm{Hsu}$ and Kazarinoff [9]. Consider the $n$-dimensional autonomous system of differential equations $\dot{x}$ $=A(\mu) x+F(x, \mu)$ where $x=\operatorname{col}\left(x_{1}, x_{2}, \cdots, x_{n}\right), F=\operatorname{col}\left(f_{1}, \cdots, f_{n}\right), F(0, \mu) \equiv 0$ and $F_{x}(0, \mu) \equiv 0$. Suppose that $f_{i}(1 \leq i \leq n)$ is a real-valued analytic function of $G x(-c, c)$ where $c>0$ and $G$ is an open connected domain in $R^{n}$. Also let $A(\mu)$ be a real, $n \times n$ matrix, analytic in $\mu$ for $\mu \in(-c, c)$ with exactly two purely imaginary eigenvalues $\pm i \beta_{0}$ at $\mu=0$ and whose continuous extensions $\alpha(\mu) \pm i \beta(\mu)$ satisfy the conditions

$$
\alpha(0)=0, \beta(0)=\beta_{0}>0,\left.\frac{d \alpha(\mu)}{d \mu}\right|_{\mu=0} \neq 0 .
$$

Under these assumptions we state

THEOREM (Hopf [8]): There is a value $\zeta_{0}>0$ such that for each $\zeta \in\left(-\zeta_{0}, \zeta_{0}\right)$ there is a periodic solution $p(t, \zeta)$, with period $T(\zeta)$, of the equation $\dot{x}=A(\mu) x+F(x, \mu)$ where $\mu$ $=\mu(\zeta)$ is analytic at $\zeta=0$ and $\mu(0)=0$. The function $p(t, \zeta)$ is analytic at $\zeta=0$ with $p(t, 0)$ $=0$ and $p(t, \zeta) \neq 0$ for sufficiently small $\zeta \neq 0$. Also $T(\zeta)$, the period of $p(t, \zeta)$, is analytic at $\zeta=0$ with $T(0)=2 \pi / \beta_{0}$. The periodic solutions exist either only for $\zeta>0$, only for $\zeta<$ 0 or only for $\zeta=0$. Furthermore, for each $L>T(0)$ there are positive numbers $a>0$ and $b$ $>0$ such that if $|\mu|<b$ then there can be no nonconstant periodic solution of period less than $L$, besides the bifurcating periodic solutions, whose orbit is entirely contained within the set $\{x|| x||<a\}$.

We apply the Hopf theory to the system (2.1)-(2.4).

For each $I \geq 0$ let $y^{\prime}=A_{\epsilon}(I) y$ denote the linearization of the system (2.1)-(2.4) around 
the steady-state solution $\pi_{I}$. The matrix $A_{\epsilon}(I)$ is defined by

$$
A_{\epsilon}(I)=\left.\left[\begin{array}{cccc}
-F_{v} & -F_{m} & -N_{n} & -F_{h} \\
\gamma_{m} m_{\infty}^{\prime} & -\gamma_{m} & 0 & 0 \\
\epsilon \gamma_{n} h_{\infty}^{\prime} & 0 & -\epsilon \gamma_{n} & 0 \\
\epsilon \gamma_{h} h_{\infty}^{\prime} & 0 & 0 & -\epsilon \gamma_{h}
\end{array}\right]\right|_{.(v, m, n, h)=\pi_{I}}
$$

From (4.1) we compute

$$
\operatorname{det}\left(A_{\epsilon}(I)\right)=\left.\epsilon^{2} \gamma_{m} \gamma_{n} \gamma_{h}\left(F_{v}+m_{\infty}{ }^{\prime}+n_{\infty}{ }^{\prime} F_{n}+h_{\infty}{ }^{\prime} F_{h}\right)\right|_{(v, m, n, h)=\pi_{I}} .
$$

A routine calculation shows that the characteristic polynomial associated with $A_{\epsilon}(I)$ is given by $p(\lambda)=\lambda^{4}+c_{1} \lambda^{3}+c_{2} \lambda^{2}+c_{23} \lambda+c_{4}$, where

$$
\begin{gathered}
c_{1}=\gamma_{m}+F_{v}+\epsilon\left(\gamma_{n}+\gamma_{n}\right), \\
c_{2}=\gamma_{m}\left(F_{v}+m_{\infty}{ }^{\prime} F_{m}\right)+\epsilon\left(\left(\gamma_{m}+F_{v}\right)\left(\gamma_{n}+\gamma_{h}\right)+\gamma_{n} n_{\infty}{ }^{\prime} F_{n}+\gamma_{h} h_{\infty}{ }^{\prime} F_{h}\right)+\epsilon^{2} \gamma_{n} \gamma_{h}, \\
c_{3}=\epsilon\left(\gamma_{m}\left(\gamma_{n}+\gamma_{h}\right)\left(F_{v}+m_{\infty}{ }^{\prime} F_{m}\right)+\gamma_{m}\left(\gamma_{n} n_{\infty}{ }^{\prime} F_{n}+\gamma_{h} h_{\infty}{ }^{\prime} F_{h}\right)\right) \\
+\epsilon^{2}\left(\gamma_{m}+F_{v}+n_{\infty}{ }^{\prime} F_{n}+h_{\infty}{ }^{\prime} F_{h}\right) \\
c_{4}=\epsilon^{2} \gamma_{m} \gamma_{n} \gamma_{h}\left(F_{v}+m_{\infty}^{\prime} F_{m}+n_{\infty}{ }^{\prime} F_{n}+h_{\infty}{ }^{\prime} F_{h}\right)
\end{gathered}
$$

with all functions appearing on the right-hand side of (4.3) being evaluated at $(v, m, n, h)$ $=\left(v_{I}, m_{\infty}\left(v_{I}\right), n_{\infty}\left(v_{I}\right), h_{\infty}\left(v_{I}\right)\right)$. Define $\Gamma_{\alpha}=[0, I(\bar{v}+\alpha)] \cup[I(\hat{v}-\alpha), I(\hat{v})]$ for each $\alpha>0$. From (2.16), Lemma 1 and assumptions (iii), (v) and (vi) it follows that there is an $\alpha_{1}>0$ such that if $0<\alpha<\alpha_{1}$ then Lemma 1 is satisfied and, in addition,

$$
c_{i}>0 \text { for all } \epsilon>0 \text { and for all } I \epsilon \Gamma_{\alpha} \text {, for } i=1,3,4 \text {. }
$$

In order to apply the Hopf theory we need to show that as I passes through a critical value, exactly two eigenvalues of $A_{\epsilon}(I)$ cross the imaginary axis nontangentially. We use the Hurwitz criterion to do this.

THEOREM (Hurwitz; [14] p. 304). The roots of the equation $\lambda^{4}+c_{1} \lambda^{3}+c_{2} \lambda^{2}+c_{3} \lambda+c_{4}$ $=0$ all have negative real parts if and only if $c_{4}>0$ and

$$
\begin{gathered}
D_{1}=c_{3}, \\
D_{2}=\operatorname{det}\left[\begin{array}{ll}
c_{2} & c_{1} \\
c_{4} & c_{3}
\end{array}\right], \\
D_{3}=\operatorname{det}\left[\begin{array}{ccc}
c_{3} & c_{4} & 0 \\
c_{1} & c_{2} & c_{3} \\
0 & 1 & c_{1}
\end{array}\right]
\end{gathered}
$$

are all positive. If $0<\alpha<\alpha_{1}$ then (4.4) implies

$$
D_{1}>0 \text { for all } \epsilon>0 \text {, and all } I \epsilon \Gamma_{\alpha} \text {. }
$$

If $\alpha_{1}$ is sufficiently small then from (2.7), (2.16), and assumptions (v) and (vi) it follows that

$$
D_{2}>0 \text { and } \quad D_{3}>0
$$


for all $\epsilon>0$, and all $I \epsilon[0, \bar{I}] \cup\left[\hat{I}, I\left(\hat{v}+\alpha_{1}\right)\right]$. Thus in this range of parameters the steady state is stable. Next, expanding out $D_{3}$ we find that

$$
D_{3}=c_{1} D_{2}-c_{3}^{2}
$$

Thus (4.4), (4.6) and (4.7) imply that $D_{3}$ is the first of $D_{2}$ and $D_{3}$ to change sign as $I$ increases past $\bar{I}$ or decreases below $\hat{I}$. In order to show that $D_{3}$ does change sign we need to define the function

$$
\hat{F}(I, \epsilon)=\frac{D_{3}(I, \epsilon)}{\epsilon}
$$

where $\epsilon>0$ and $I \in \Gamma_{\alpha_{1}}$.

A straightforward algebraic calculation shows than an $\epsilon$ can be factored out of $D_{3}$ and thus our assumptions imply that $\bar{F}$ is well defined and continuously differential for $\epsilon \geq 0$ and $I \in \Gamma_{\alpha_{1}}$. Next, from assumptions (ii), (iii), (v), (vi) and (vii) it follows that

$$
\begin{gathered}
\bar{F}(\bar{I}, 0)=\bar{F}(\hat{I}, 0)=0, \\
\frac{\partial \bar{F}}{\partial I}(\bar{I}, 0)=\left[\left.\left.\left(\left(\gamma_{m}+F_{v}\right) \gamma_{m}{ }^{2}\left(\gamma_{m} n_{\infty}{ }^{\prime} F_{n}+\gamma_{n} h_{\infty}^{\prime} F_{h}\right) \frac{d b}{d v}\right]\right|_{v=\bar{v}}\left(\frac{d v_{I}}{d I}\right)\right|_{I=I}<0,\right. \\
\frac{\partial \bar{F}}{\partial \epsilon}(\bar{I}, 0)=\left(\left(\gamma_{m}+F_{v}\right)^{2} \gamma_{m}\left(\gamma_{n}{ }^{2} n_{\infty}{ }^{\prime} F_{n}+\gamma_{h}{ }^{2} h_{\infty}{ }^{\prime} F_{n}\right)\right. \\
\left.+\gamma_{m} F_{v}\left(\gamma_{n} n_{\infty}{ }^{\prime} F_{n}+\gamma_{h} h_{\infty}^{\prime} F_{h}\right)^{2}\right)\left.\right|_{v=\bar{v}}>0 .
\end{gathered}
$$

Similarly,

$$
\frac{\partial \bar{F}}{\partial I}(\hat{I}, 0)>0,
$$

and

$$
\frac{\partial \bar{F}}{\partial \epsilon}(\hat{I}, 0)>0
$$

From (4.10) and the implicit function theorem it follows that there are open intervals $U_{1}$ and $W_{1}$ containing $\epsilon=0$ and $I=I I$, respectively, such that for each $\epsilon$ in $U_{1}$ there is a unique $I_{\epsilon}$ which satisfies

$$
\bar{F}\left(I_{\epsilon}, \epsilon\right)=0 .
$$

Also, $I_{\epsilon}$ is continuously differentiable over $U_{1}$ and (4.14), (4.10) and (4.11) imply that

$$
\frac{d I_{\epsilon}}{d \epsilon}=\left.\left(-\frac{\partial \bar{F}}{\partial \epsilon} / \frac{\partial \bar{F}}{\partial I}\right)\right|_{\left(I_{\epsilon}, \epsilon\right)}>0
$$

if $\epsilon>0$ is sufficiently small.

Similarly there are neighborhoods $U_{2}$ and $W_{2}$ of $\epsilon=0$ and $I=\hat{I}$, respectively, such that for each $\epsilon$ in $U_{2}$ there is a unique value $I^{\epsilon}$ in $W_{2}$ which satisfies

$$
\bar{F}\left(I^{\epsilon}, \epsilon\right)=0
$$

Also, $I^{\epsilon}$ is continuously differentiable with respect to $\epsilon$ in $U_{2}$ and (4.14), (4.12) and (4.13) 
imply

$$
\frac{d I^{\epsilon}}{d \epsilon}=\left.\left(-\frac{\partial \bar{F}}{\partial \epsilon} / \frac{\partial \bar{F}}{\partial I}\right)\right|_{\left(I^{\epsilon}, \epsilon\right)}<0
$$

if $\epsilon>0$ is sufficiently small.

The implicit function theorem also guarantees that

$$
\lim _{\epsilon \rightarrow 0} I^{\epsilon}=\bar{I} \text { and } \lim _{\epsilon \rightarrow 0} I^{\epsilon}=\hat{I} .
$$

Thus, let $\alpha$ satisfy Lemma 1, (4.4) and (4.6), and define $U=U_{1} \cap U_{2}$. Let $\epsilon_{\alpha}=\sup U$. Then the previous analysis indicates that for each positive $\epsilon<\epsilon_{\alpha}$ there are two values $I_{\epsilon}$ and $I^{\epsilon}$ such that $\bar{I}<I_{\epsilon}<I(\bar{v}+\alpha) \leq I(\hat{v}-\alpha)<I^{\epsilon}<\hat{I}$ and

$$
\begin{gathered}
D_{1}>0 \text { for all } I \in\left[\bar{I}, I_{\epsilon}\right] \cup\left[I^{\epsilon}, \hat{I}\right], \\
D_{2}>0 \text { for all } I \in\left[\bar{I}, I_{\epsilon}\right] \cup\left[I^{\epsilon}, \hat{I}\right], \\
D_{3}>0 \text { for all } I \in\left[\bar{I}, I_{\epsilon}\right] \cup\left[I^{\epsilon}, \hat{I}\right], \\
D_{3}\left(I_{\epsilon}, \epsilon\right)=D_{3}\left(I^{\epsilon}, \epsilon\right)=0 .
\end{gathered}
$$

Thus (4.19) together with the Hurwitz theorem implies that for each $\epsilon$ in $\left(0, \epsilon_{\alpha}\right)$ there are at least two purely imaginary eigenvalues of $A_{\epsilon}\left(I_{\epsilon}\right)$ and $A_{\epsilon}\left(I^{\epsilon}\right)$. If either $A_{\epsilon}\left(I_{\epsilon}\right)$ or $A_{\epsilon}\left(I^{\epsilon}\right)$ has zero as an eigenvalue then $c_{4}=0$, contradicting (4.4). If all four eigenvalues of either $A_{\epsilon}\left(I_{\epsilon}\right)$ or $A_{\epsilon}\left(I^{\epsilon}\right)$ are purely imaginary then their sum is zero; hence $c_{1}=0$, contradicting (4.4). Thus there are exactly two purely imaginary eigenvalues $\pm i B_{\epsilon}$, and $\pm i B^{\epsilon}$ of $A_{\epsilon}\left(I_{\epsilon}\right)$ and $A_{\epsilon}\left(I^{\epsilon}\right)$ respectively. Let $\gamma_{\epsilon}(I)$ and $\gamma^{\epsilon}(I)$ denote the complex-valued $C^{1}$ extensions of $i B_{\epsilon}$ and $i B^{\epsilon}$ respectively. In order to apply the Hopf theory and complete the proof of our theorem we must show that

$$
\left.\operatorname{Re}\left(\frac{d \gamma_{\epsilon}(I)}{d \epsilon}\right)\right|_{I=I_{\epsilon}} \neq 0 \quad,\left.\quad \operatorname{Re}\left(\frac{d \gamma^{\epsilon}(I)}{d I}\right)\right|_{I=I^{*}} \neq 0
$$

The proof of (4.20) follows in exactly the same manner as that given in previous work by Troy $([12,13])$ where it was shown that there exists at least one set of values $(I, \epsilon)$ where a bifurcation of periodic solutions takes place. Thus, for brevity we omit the details.

Finally, make the transformation $I=I_{\epsilon}+\mu$ or $I=I^{\epsilon}+\mu$ in (2.1). Then as $\mu$ passes through zero it is clear that all conditions of the Hopf theorem are satisfied and the proof of our theorem easily follows.

\section{References}

[1] K. S. Cole, H. A. Antosiewicz, and P. Rabinowitz, Automatic computation of nerve excitation, SIAM J. Appl. Math. 3, 153-172 (1955)

[2] Cooley, Dodge and Cohen, J Cellular Comp. Phys. 66, 99-110 (1965)

[3] R. FitzHugh, Thresholds and plateaus in the Hodgkin-Huxley nerve equations, J. Gen. Physiology 43, 867896 (1960)

[4] R. FitzHugh and H. A. Antosiewicz, Automatic computation of nerve excitation: detailed corrections and additions, SIAM. J. Appl. Math. 7, 447-458 (1959)

[5] S. Hastings, Travelling wave solutions of the Hodgkin-Huxley equations, Arch. Rat. Mech. A nal. 60, 229-257 (1975)

[6] O. Hauswirth, D. Noble, and R. W. Tsien, The mechanism of oscillatory activity at low membrane potentials in cardiac purkinje fibres, J. Physiol. 200, 255-265 (1969)

[7] A. L. Hodgkin and A. F. Huxley, A quantitative description of membrane current and its application to conduction and excitation in nerve, J. Physiol. 117, 500-544 (1952) 
[8] E. Hopf, Abzweigung einer periodischen Losung von einer stationaren Losung eines differential Systems, Ber. Verh. Sachs. Akad. Wiss. Leipsig. Math.-Nat. 94, 1-22 (1942)

[9] I. D. Hsu and N. D. Kazarinoff, An applicable Hopf bifurcation formula and instability of small periodic solutions in the field Noyes model, JMAA 55, 61-89 (1976)

[10] R. E. McAllister, D. Noble, and R. W. Tsien, Reconstruction of the electrical activity of cardiac purkinje fibres, J. Phys. 251, 1-59 (1975)

[11] N. H. Sabah and R. A. Spangler, Repetitive response of the Hodgkin-Huxley model for the squid giant axon, J. Theor. Biol. 29, 155-171 (1970)

[12] W. C. Troy, doctoral dissertation, SUNY Buffalo, 1974

[13] W. C. Troy, Oscillation phenomena in the Hodgkin-Huxley equations, Proc. Roy. Soc. Edinburgh 74 A, 299$310(1976)$

[14] J. Uspensky, Theory of equations, New York, 1948 